\title{
Anterior Abdominal Wall Endometriosis Following Caesarean Section: Two Rare Cases
}

\author{
Meryem Belmajdoub, \\ Sofia Jayi, \\ Fatima Zehra Fdili Alaoui, \\ Hekmat Chaara, \\ Moulay Abdelilah Melhouf, \\ Service de Gynécologie Obstétrique 2, \\ Centre Hospitalier Universitaire HASSAN 2, Fès, Maroc
}

Doi:10.19044/esj.2019.v15n30p105 URL:http://dx.doi.org/10.19044/esj.2019.v15n30p105

\begin{abstract}
Parietal endometriosis is a rare clinical entity, whose pathophysiology remains unclear. It occurs most often after gynecological or obstetrical surgery. The clinical picture is relatively unspecific. Medical imaging is not very contributive. Only histological examination of the operative specimen can confirm the diagnosis. Surgical treatment must be wide enough to prevent recurrence. We report two cases of abdominal wall endometriosis occurring on caesarean section scar. Through these two cases, we will highlight the characteristics of this pathology including prognosis, which will help the practitioners in understanding the role of diagnosis and early management of this entity and its prevention during gynecological or obstetrical surgery.
\end{abstract}

Keywords: Endometriosis, Abdominal Wall, Diagnosis, Treatment, Prognosis, Prevention

\section{Introduction}

Endometriosis is described by the presence of functional endometrial tissue outside the uterine cavity (Audebert, 2013). Its most frequent localization is in the internal genital organs, but other extra pelvic locations are described notably in the lungs, intestines, urinary tract, central nervous system, perineum and abdominal wall. At the level of the abdominal wall its occurrence in the scars of gynecological or obstetrical surgery is rare (0.03$0.4 \%$ ) (Picod et al, 2006). The clinical presentation is relatively unspecific. Medical imaging is not very contributive. Only histological examination of the operative specimen can confirm the diagnosis. Surgical treatment must be wide enough to prevent recurrence. Given the rarity and the unusual nature of 
this localization, we report two new cases of endometriosis of the abdominal wall, through which and the light of a review of the literature we will emphasize the importance of diagnosis and early management of this entity and its prevention during each gynecological surgery.

\section{Observations}

\section{Case 1:}

This is a 41-year-old patient, third parity and gesture, who twice benefited from a caesarean section, the last one dating back to 6 years, who complains of pain in the caesarean section scar with development of a mass increasing in size with associated pain punctuated by the menstrual cycle. The abdominal examination shows a mass of $7 \mathrm{~cm} / 6 \mathrm{~cm}$ fixed to the deep plane sitting above the left end of pfannenstiel scar. Ultrasonography of the soft tissues showed the presence of a parietal mass of multi-lobed tissue, presenting an arteriovenous central vascularization to the color Doppler, measuring 65/58 $\mathrm{mm}$, making discuss an endometriotic mass or a tumor of the soft parts. Pelvic MRI shows a mass of the left rectus muscle presenting with T2 hypersignal and T1 hypersignal, heterogeneous, not cleared after removal of the fat siginal, significantly and heterogeneously improved after Gadolinium injection delimiting liquefaction zones and measuring $63 * 24 * 79 \mathrm{~mm}$ with presence of signs of deep pelvic endometriosis (Figure 1), based on this clinical and radiological picture the diagnosis of parietal endometriosis was retained and in this sense a wide excision of the mass was performed (Figure 2). In view of the importance of parietal loss parietoplasty was performed by interposition of a polypropylene plate. Anatomopathological examination of the resection specimen revealed that it was a site of endometriosis of the abdominal wall. Immediate postoperative outcomes were simple. The patient reported the disappearance of dysmenorrhea in the weeks following surgery. A control at 3 months and then every 6 months was carried out not objecting to any recurrence over a 12-month follow-up. 


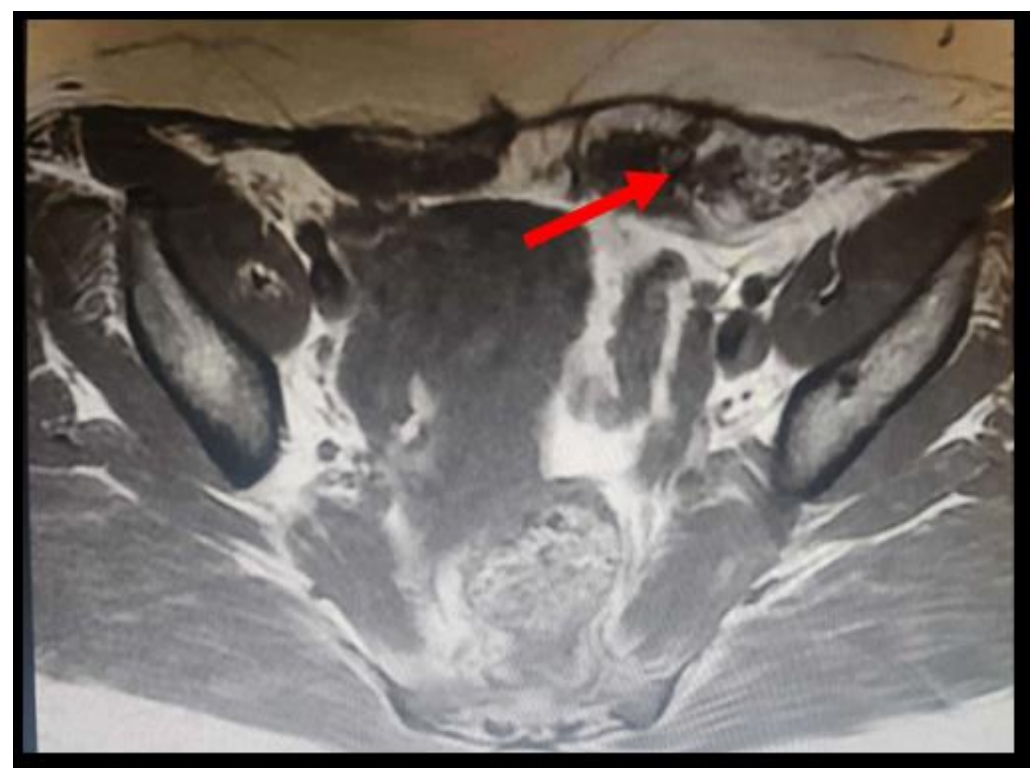

Figure 1:

Case 1: Pelvic MRI

Axial section showing the mass in contact with the left rectus muscle of the abdomen

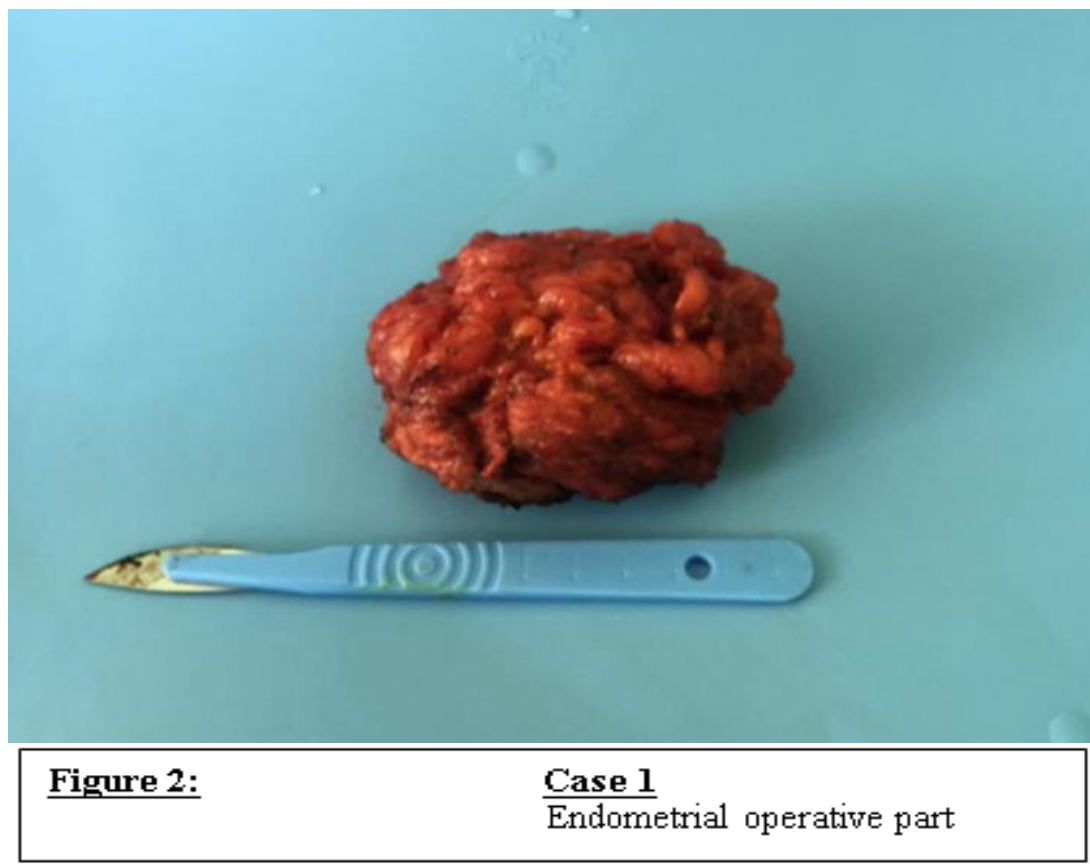




\section{Case 2:}

This is a 43 year-old patient, second gesture and second parity, with no pathological antecedents except for a doubly scarred uterus whose last Caesarean section dates back to 5 years. The beginning of the symptomatology goes back to 3 years by the appearance of a mass increasing size associated with hypogastric pains rhythmized by the menstrual cycle and for which the abdominal examination shows a mass of $4 \mathrm{~cm} / 3 \mathrm{~cm}$ fixed in the deep plane sitting above the left end of the pfannenstiel scar without palpable lymphadenopathy (Figure 1). Ultrasonography of the soft tissues showed the presence of a parietal mass of multi-lobed tissue, presenting an arteriovenous central vascularization with color Doppler, measuring 22/21 mm (Figure 2), discussing an endometriotic mass or a soft tissue tumor. A pelvic MRI shows a subcutaneous parietal nodule of the left iliac fossa coming into contact with the superficial aponeurosis of $20 / 26 \mathrm{~mm}$ in diameter, with speculative contours, surrounded by a hypo T2 signal, highlighted after contrast in a heterogeneous and important way and not showing an extension at the muscular level with regard, whose appearance is in favor of parietal endometriosis of the left iliac fossa coming into contact with the superficial aponeurosis (Figure 3). Hence the decision of excision of the lesion with discovery of a hard mass of $3 / 2 \mathrm{~cm}$, sub fascial attached to the rectus abdominis muscle. The pathological study confirmed the diagnosis of parietal endometriosis. The postoperative follow-up was simple with a good evolution and a 6-month follow-up without recurrence of mass or pain.

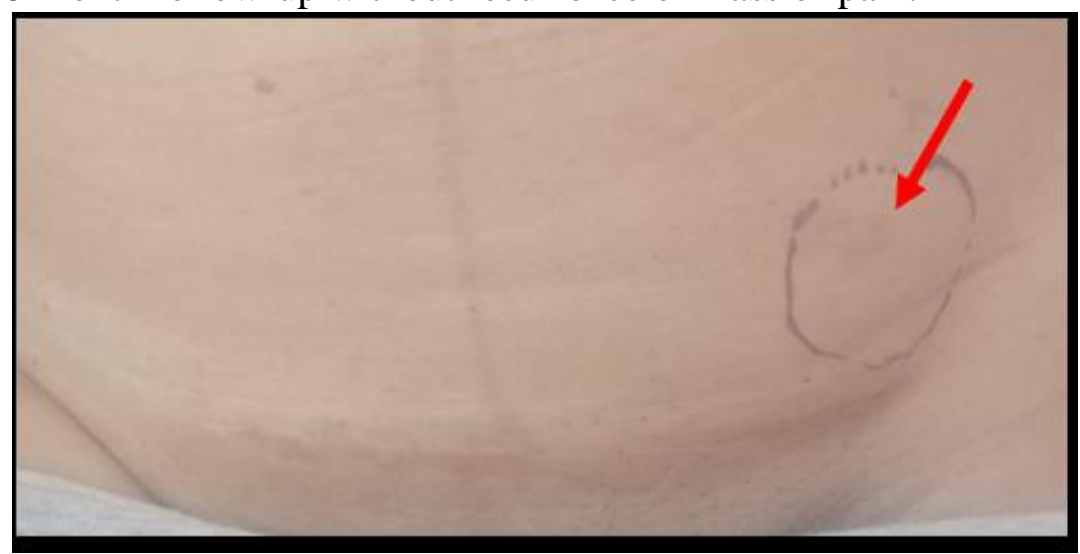

Figure 1:

Case 2: clinical examination

The location of the lesion above the left end of the pfannenstial scar 


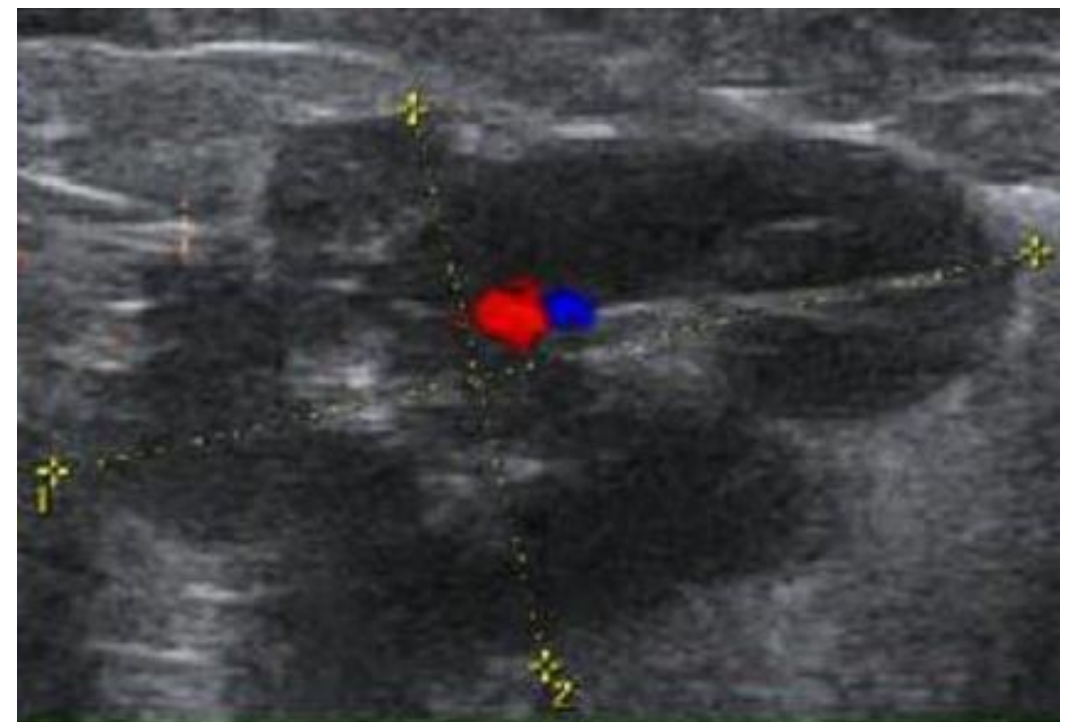

Figure 2:

Case 2: Ultrasound of soft parts

Ultrasound showing a heterogeneous hypoechoic image with irregular contours, and vascularized with color Doppler

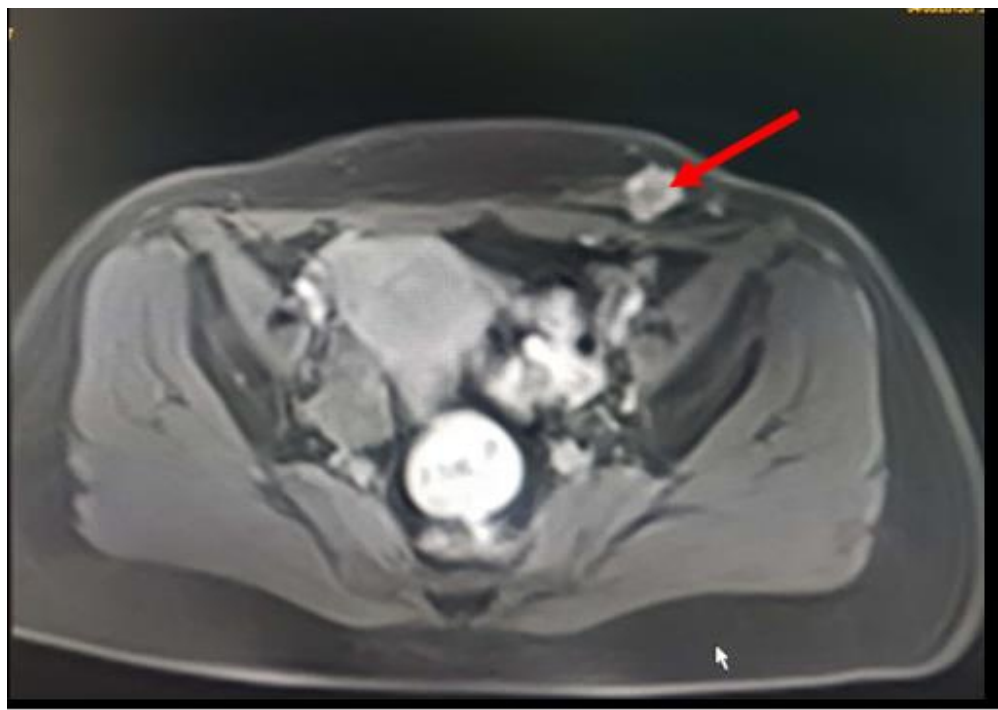

Figure 3:

Case 2: Pehic MRI

Axial section showing the mass in contact with the left rectus muscle of the abdomen

\section{Discussion}

Parietal endometriosis is a rare pathology whose initial diagnosis is not always easy. It represents $0.03 \%$ to $2 \%$ of extra-genital endometriosis (Picod et al, 2006), (Hafidi et al, 2011). However this frequency is much higher than that observed after conventional gynecological surgery (Victory et al, 2007). 
In the vast majority of cases, they have cicatricial localization following a surgical procedure, whether by laparoscopic, on the various insertion sites of the trocars or by laparotomy (Hafidi et al, 2011), (Sirito et al, 2005), or even after amniocentesis (Hafidi et al, 2011), (Patil et al, 2014). All gynecological interventions predispose to this pathology but those involving hysterectomy and especially uterine morcellation expose to an increased risk (Sirito et al, 2005). It should be noted that Rani et al showed that endometriosis was particularly common after hysterotomy in the 2nd or early 3rd trimester of pregnancy. They suggest that the endometrium of early pregnancy may be more easily transplanted than endometrium of late pregnancy. However, some cases of primary parietal endometriosis have been reported mainly in the umbilicus, right muscles and inguinal region secondary to vascular or lymphatic dissemination (Sirito et al, 2005). While cicatricial localization is thought to be due to the direct implantation of endometrial cells followed by a secondary inflammation probably induced by immunological factors and by a high potential of development of these endometrial cells to nonepithelialized zones (Jayi et al, 2008).

The diagnosis of parietal endometriosis can be easy to evoke in typical cases, but sometimes it is more difficult. Classically parietal endometriosis is manifested by a triad as is the case with our patients: firm palpable mass, with variable size, adherent to the fascia, with cyclical pain and catamenial exacerbation. The time of appearance remains very variable from 2 months to 15 years after the surgical procedure and some are even seen after menopause by reactivation of the foci under the effect of hormone replacement therapy or in the presence of a estrogen-secreting tumor. Some differential diagnoses may be discussed clinically: hematoma, abscess, hernia, evisceration, and tumors when the mass is distant from the scar; as well as the scar granuloma and evisceration if the mass is next to the scar (Sirito et al, 2005), (Jayi et al, 2008).

Once the parietal endometriosis is suspected, the medical imaging contribution is of less importance, the first examination to be requested remains the echography with Doppler color, which plays an important role in the diagnostic orientation and the preoperative assessment. It is most often a hyper-vascularized hypo-echogenic image as it has been found in our cases, mixed or fluid, whose contours are spiculated but sometimes it can be hyperechogenic according to: the phase of the menstrual cycle, the distribution between the elements of the glandular stroma and the importance of the inflammatory reaction. MRI is the key examination showing the particular signal of bleeding in an endometrioma, iso or hypo-intense nodule in T1 and T2 punctuated with hyper-intense foci in T1 and T2 (Khlifi et al, 2012).

The aspiration puncture or percutaneous biopsy may allow the diagnosis to be made or confirmed before considering a surgical treatment (Khlifi et al, 2012), 
but it should be avoided because of the risk of dissemination along the puncture route (Hafidi et al, 2011). Confirmation of the diagnosis is based on the histological study of the excised material when it shows the presence of endometrial glands, however in some cases, the differential diagnosis is discussed with adenocarcinoma or metastases of adenocarcinoma (Khlifi et al, 2012). This excision is at the same time the gold standard of the treatment and should pass well off the lesion, at least $5 \mathrm{~mm}$ in the healthy zone, with placement of prosthetic plaque in the event of significant fascial defect (Jayi et al, 2008), (CNGOF, 2013). Post-operative medical treatment with LHRH or DANAZOL analogue has been proposed, but its benefit has not yet been demonstrated. $\mathrm{CO} 2$ laser photocoagulation has also been reported to be effective but remains uncommon (Jayi et al, 2008).

Recurrences are not uncommon, ranging from 10 to $15 \%$. The recurrence rate is correlated with the size and depth of the lesion. A nonrandomized study concluded that preoperative treatment appears to reduce the rate of recurrence (CNGOF, 2013). In addition, some cases of cancer of endometriosis of the wall have been reported in the literature, which justifies the systematic excision of such lesions (Sergent et al, 2006). Compared to ovarian cancer on endometriosis, the prognosis for this complication of an abdominal scar is rather bleak. In the literature, the survival rate reaches $57 \%$ with a short follow-up of 20 months and the most common histological form is clear cell carcinoma, followed by endometrioid carcinoma (Sergent et al, 2006).

Prevention in case of laparotomy is based on the abundant washing of the abdominal cavity and the scar at the end of the procedure as well as the change of gloves in the parietal closure time, whereas in laparoscopy, the extraction of the surgical specimens in a protective bag and the abundant washing of the pelvic cavity should be systematic. Thus, these measures belong to a good surgical practice although their benefit has never been demonstrated (CNGOF, 2013), (Sergent et al, 2006).

\section{Conclusion}

Parietal endometriosis is a pathology certainly rare but whose diagnosis must be evoked in women who have benefited from open gynecological or obstetrical surgery and have a mass of the wall with cyclical pain, in the near or distant suites of this surgery. This should always lead to extensive excision because of the risk of cancer whose prognosis is considered dark. 


\section{References:}

1. Audebert A. Les endométrioses iatrogènes de la femme avant la ménopause: principaux enjeux. Gynécologie Obstétrique \& Fertilité 2013;41:322-327.

2. Picod G, Boulanger L, Bounoua F, Leduc F, Duval G. Endométriose pariétale sur cicatrice de césarienne: à propos de 15 cas. Gynécologie Obstétrique \& Fertilité 2006;34(1):8-13.

3. Hafidi R, Kouach J, Yakka M, Salek G, Oukabli M, Moussaoui RD, Dehayni M. L'endométriose cicatricielle de la paroi abdominale. Imagerie de la Femme. 2011;21(3):125-127.

4. Victory R, Diamond MP, Johns DA. Villar's nodule: a case report and systematic literature review of endometriosis externa of the umbilicus. J Minim Invasive Gynecol. 2007 Jan-Feb;14(1):23-32.

5. Sirito R, Puppo A, Centurioni MG, Gustavino C. Incisional hernia on the 5-mm trocar port site and subsequent wall endometriosis on the same site: a case report. Am J Obstet Gynecol. 2005 Sep;193(3 Pt 1):878-80.

6. Patil N, Kumar V, Gupta A. Scar endometriosis- a sequel of caesarean section. J Clin Diagn Res. 2014 Apr;8(4):FD09-10

7. Jayi S, Bouguern H, Bouchikhi C, Amhager N, Chaara H, Melhouf MA, Banani A. Greffe endométriosique post-obstétricale, à propos de 2 cas. Médecine du Maghreb. 2008;154:21-25.

8. Khlifi A, Kouira M, Memmi A, Ziadi S, Labidi I, et al. Endométriose pariétale. Imagerie de la Femme. 2012;22(3):156-161.

9. CNGOF. Prise en charge de l'endométriose - Recommandations pour la pratique clinique. Mises à jour en gynécologie obstétrique. Disponible sur: http://www.cngof.asso.fr/D_TELE/RPC_endometriose.pdf. Accédé le 2 Juin 2013.

10. Sergent F, Baron M, Le Cornec JB, Scotté M, et al. Transformation maligne d'une endométriose pariétale: un nouveau cas. Journal de Gynécologie Obstétrique et Biologie de la Reproduction. 2006;35(2):186-190 